\title{
2020 Korean Guidelines for Cardiopulmonary Resuscitation. Part 1. Update process and highlights
}

\section{Sung Oh Hwang ${ }^{1}$, Kyoung-Chul Cha ${ }^{1}$, Woo Jin Jung ${ }^{1}$, Young-II Roh ${ }^{1}$, Tae Youn Kim ${ }^{1}$, Sung Phil Chung ${ }^{2}$, Young-Min Kim ${ }^{3}$, June Dong Park ${ }^{4}$, Han-Suk Kim ${ }^{4}$, Mi Jin Lee ${ }^{5}$, Sang-Hoon $\mathrm{Na}^{6}$, Gyu Chong Cho Ai-Rhan Ellen $\mathrm{Kim}^{8}$; on behalf of the Steering Committee of the 2020 Korean Guidelines for Cardiopulmonary Resuscitation and Emergency Cardiovascular Care}

\author{
'Department of Emergency Medicine, Yonsei University Wonju College of Medicine, Wonju, Korea \\ ${ }^{2}$ Department of Emergency Medicine, Yonsei University College of Medicine, Seoul, Korea \\ ${ }^{3}$ Department of Internal Medicine, The Catholic University of Korea College of Medicine, Seoul, Korea \\ ${ }^{4}$ Department of Pediatrics, Seoul National University College of Medicine, Seoul, Korea \\ ${ }^{5}$ Department of Emergency Medicine, Kyoungbook University College of Medicine, Daegu, Korea \\ ${ }^{6}$ Department of Internal Medicine, Seoul National University College of Medicine, Seoul, Korea \\ ${ }^{7}$ Department of Emergency Medicine, Hallym University College of Medicine, Seoul, Korea \\ ${ }^{8}$ Department of Pediatrics, Ulsan University College of Medicine, Seoul, Korea
}

elSSN: 2383-4625

Received: 7 March 2021

Revised: 7 March 2021

Accepted: 22 March 2021

Correspondence to: Sung Oh Hwang Department of Emergency Medicine, Yonsei University Wonju College of Medicine, 20 Ilsan-ro, Wonju 26426, Korea

E-mail: shwang@yonsei.ac.kr ORCID

https://orcid.org/0000-0003-4585-3181

\section{BACKGROUND}

Out-of-hospital cardiac arrest (OHCA) is one of the growing medical issues in developing and developed countries. The incidence of OHCA ranges from 24 to 186 per 100,000 population, and varies according to ethnic group, country, and region. ${ }^{1}$ The annual incidence of OHCA in Korea increased from 21,905 (44.3 per 100,000 population) in 2008 to 30,539 (59.5 per 100,000 population) in 2018. ${ }^{2}$ Considering the demographic structure of Korea, which is entering an aging society, the incidence of cardiac arrest is likely to increase. The survival rate of victims of cardiac arrest is affected by various factors such as the demographic and social characteristics of each country or community, the effectiveness of the emergency medical system (EMS), the rate of bystander cardiopulmonary resuscitation (CPR), and the public access defibrillation program. Although 60 years have passed since the introduction of modern CPR, the survival rate of victims of cardiac arrest remains low. The survival-to-discharge rate of OHCA in Korea increased from $3.0 \%$ in 2008 to $8.6 \%$ in 2018, and the rate of favorable neurological outcomes increased from 0.9\% in 2008 to $5.1 \%$ in 2018. ${ }^{2}$ The survival rate of OHCA in the United States, Europe, and Japan, where research on cardiac arrest started early, CPR education was provided to citizens and public access defibrillation programs were implemented, is over $10 \% .^{3-5}$

Cardiac arrest occurs in locations outside the hospital such as home, street, public places, or sports facilities. Due to the nature of OHCA, witnesses who are not medical personnel play an important role in the process of rescuing a victim of cardiac arrest. The survival of victims of OHCA is affected by the effectiveness of out-of-hospital rescue activities, including the recognition of cardiac arrest by witnesses, bystander CPR, response time of the EMS, and on-site use of

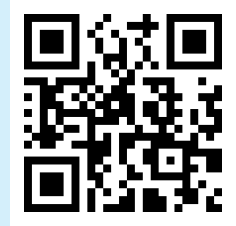

How to cite this article:

Hwang SO, Cha KC, Jung WJ, Roh YI, Kim TY, Chung SP, Kim YM, Park JD, Kim HS, Lee MJ, $\mathrm{Na} \mathrm{SH}, \mathrm{Cho} \mathrm{GC}$, Kim AE; on behalf of the Steering Committee of the 2020 Korean Guidelines for Cardiopulmonary Resuscitation and Emergency Cardiovascular Care. 2020 Korean Guidelines for Cardiopulmonary Resuscitation. Part 1. Update process and highlights. Clin Exp Emerg Med 2021;8(S):S1-S7. https://doi. org/10.15441/ceem.21.021

This is an Open Access article distributed under the terms of the Creative Commons Attribution Non-Commercial License (https:// creativecommons.org/licenses/by-nc/4.0/). 
an automated external defibrillator (AED). ${ }^{6}$ To effectively treat cardiac arrest, it is important that people learn how to handle a cardiac arrest situation, how to perform CPR and use an AED, and how to carry out essential procedures on a victim of cardiac arrest. Therefore, each country or community has a variety of systems in place for effective cardiac arrest treatment and policies to enhance the ability of their citizens to cope with cardiac arrest by educating them on CPR.

The CPR guidelines are a set of medical recommendations for cardiac arrest treatment based on scientific evidence. The International Liaison Committee on Resuscitation (ILCOR), a leading international organization that establishes CPR guidelines, publishes the International Consensus on Cardiopulmonary Resuscitation and Emergency Cardiovascular Care Science with Treatment Recommendations (CoSTR) on a 5-year cycle, and has been releasing updates annually since $2015 .^{7-12}$ Based on the results of the ILCOR's review of scientific evidence, each country or community updates or revises their CPR guidelines as appropriate for that country or community, taking into account the epidemiology of cardiac arrest, the EMS, the medical environment, laws, and socio-cultural background of the country or community.

Korea has been updating its CPR guidelines every five years since the first CPR guidelines were established in 2006 by the Korean Association of Cardiopulmonary Resuscitation. ${ }^{13}$ This article describes the update process and major changes in the 2020 Korean CPR guidelines.

\section{Update process of the 2020 Korean CPR guidelines}

The 2020 CPR guidelines include adult basic life support (BLS), adult advanced life support (ALS), post-cardiac arrest care (PCC), pediatric life support (PLS), neonatal resuscitation (NR), and education/implementation (EI). Environment and chain of survival for cardiac arrest is described as a new chapter (Table 1).

A steering committee and taskforces for each field (BLS, ALS, PCC, PLS, NR, and El) were formed for the revision of the guidelines. The members of the taskforces and the evidence reviewers consisted of experts recommended by 15 academic organizations related to the CPR guidelines. The taskforce members and evidence reviewers received instructions on the methodology for revising the guidelines, including literature search, systemic review and meta-analysis, the Grading of Recommendations Assessment, Development, and Evaluation (GRADE) method, and clinical practice guideline development methodologies. To review the evidence, each taskforce developed 'population, intervention, com-

Table 1. Topics of the 2020 Korean Guidelines for Cardiopulmonary Resuscitation

\begin{tabular}{|c|c|}
\hline Discipline & Topic \\
\hline $\begin{array}{l}\text { Environment for cardiac arrest survival } \\
\text { and the chain of survival }\end{array}$ & $\begin{array}{l}\text { Prevention of cardiac arrest, CPR education, cardiac arrest treatment system, chain of survival for } \mathrm{OHCA} \text {, chain of survival for } \\
\text { IHCA }\end{array}$ \\
\hline Post-cardiac arrest care & $\begin{array}{l}\text { Post-cardiac arrest care algorithm, post-cardiac arrest syndrome, comprehensive post-cardiac arrest care, treatment for } \\
\text { neurological recovery, target temperature management, withdrawal of life-sustaining treatment, prognostication of neurological } \\
\text { outcomes, organ donation after cardiac arrest, cardiac arrest center, and evaluation and rehabilitation of cardiac arrest } \\
\text { survivors }\end{array}$ \\
\hline Pediatric basic life support & $\begin{array}{l}\text { Pediatric chain of survival, algorithms for pediatric } \mathrm{OHCA} \text { and IHCA, chest compression, airway management, artificial } \\
\text { ventilation, compression-only CPR, and treatment of foreign body airway obstruction }\end{array}$ \\
\hline Neonatal resuscitation & $\begin{array}{l}\text { Physiologic response before and after birth, first step, positive pressure ventilation, chest compression, drug therapy, } \\
\text { post-resuscitation care, withholding or withdrawing resuscitation, and neonatal resuscitation education program }\end{array}$ \\
\hline Education and implementation & $\begin{array}{l}\text { Core contents and principles of resuscitation education, new educational modalities, re-training periods, quality assessment of } \\
\text { CPR education, rapid response team, roles of the emergency dispatcher, rules for termination of resuscitation, and community } \\
\text { strategies for CPR education and implementation }\end{array}$ \\
\hline
\end{tabular}

CPR, cardiopulmonary resuscitation; OHCA, out-of-hospital cardiac arrest; IHCA, in-hospital cardiac arrest; EMS, emergency medical system; COVID-19, coronavirus disease 2019. 
parator, outcome' (PICO) questions for items that required revision. Two evidence reviewers were assigned to each PICO question. After the evidence review process was complete, the reviewers reported a draft of the K-COSTR (Korean Consensus on Science and Treatment Recommendation). The results of the evidence review were approved by each taskforce and posted on the website of the Korean Association of Cardiopulmonary Resuscitation (http://www.kacpr.org/) for an online consensus conference and public hearing. The writing members of each taskforce wrote the 2020 Korean CPR guidelines based on the results of the evidence review (Fig. 1).

The evidence reviews of the PICOs were conducted in three ways. For the PICOs reviewed by ILCOR, a PICO for which no new literature was searched was reviewed by adaptation, whereas a PICO for which new literature was searched was evaluated in the way of a hybrid review. PICOs not reviewed by ILCOR were assessed in the manner of a de novo review. One hundred and thirteen PICOs, including 28 from the BLS taskforce, 19 from ALS, 15 from
PCC, 24 from PLS, 10 from NR, and 17 from El, were reviewed. PubMed (https://pubmed.ncbi.nlm.nih.gov/), Embase (https://www. embase.com), The Cochrane Central Register of Controlled Trials (https://www.cochranelibrary.com), Education Resources Information Center (https://eric.ed.gov/), and KoreaMed (https://koreamed. org/) were utilized for literature search. Only clinical research papers were considered in the literature review. Based on the results of the literature search, either a meta-analysis or scoping review was conducted. Certainty of evidence was evaluated using the GRADE method. ${ }^{14}$ The evidence reviewers wrote a draft of the Korean evidence summary and treatment recommendations. The draft contained the evidence summary, the reviewer's comments, treatment recommendations, and related references. The level of evidence and the class of recommendation were based on the definitions developed by the American Heart Association. ${ }^{15}$ Level A denotes high quality evidence from randomized controlled trials (RCTs) or meta-analyses of high quality RCTs. Level B-R (randomized) denotes moderate quality evidence from RCTs or meta-anal-

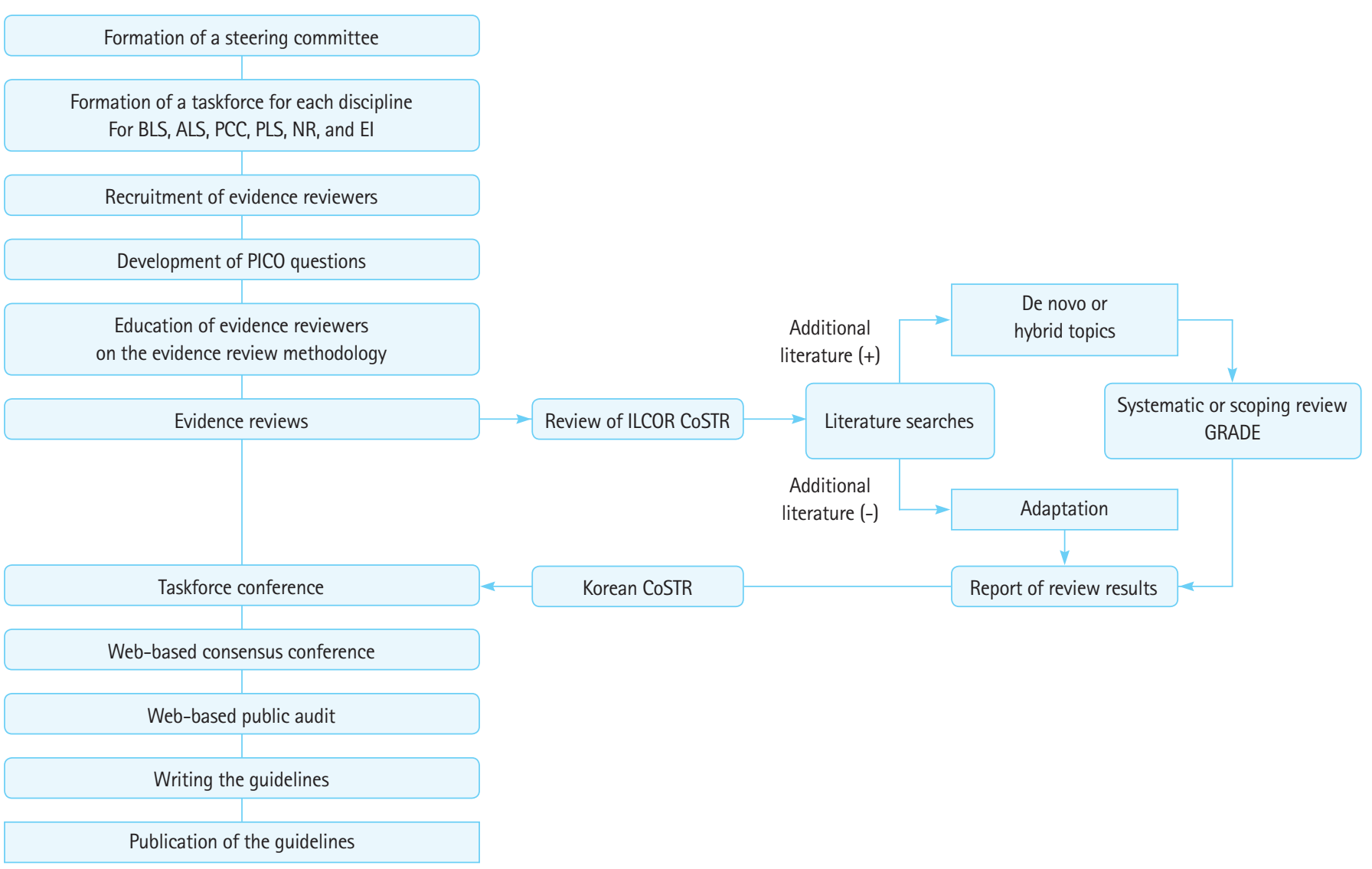

Fig. 1. The process of the guideline update. BLS, basic life support; ALS, advanced life support; PCC, post-cardiac arrest care; PLS, pediatric life support; $\mathrm{NR}$, neonatal resuscitation; El, education and implementation; PICO, population, intervention, comparator, outcome; ILCOR, International Liaison Committee on Resuscitation; CoSTR, Consensus on Science and Treatment Recommendations; GRADE, Grading of Recommendations Assessment, Development and Evaluation. 
yses of moderate quality RCTs. Level B-NR (nonrandomized) denotes moderate quality evidence from non-RCTs, observational studies, or registry studies, or meta-analyses of such studies. Level C-LD (limited data) denotes low quality evidence from observational or registry studies or meta-analyses of such studies. Level C-EO (expert opinion) denotes consensus of expert opinion. The class of recommendations was judged based on direction (benefit or harm) and strength (strong or weak) according to the GRADE method, and was divided into the three categories used by the American Heart Association. ${ }^{14,15}$ Class I indicates that the benefit of the treatment or intervention is very high relative to the risk (it is appropriate for most physicians to administer the treatment or intervention to most patients). Class Ila indicates that the treatment or intervention is generally useful (it is appropriate for most physicians to perform the treatment or intervention with some exceptions). Class Ilb indicates that the treatment or intervention has a positive effect, but the evidence is not clear. Class III (no benefit) indicates that the treatment or intervention has no benefit. Class III (harm) indicates that the treatment or intervention is harmful.

\section{HIGHLIGHTS OF THE 2020 KOREAN GUIDELINES FOR CPR}

The major changes in the 2020 Korean guidelines for CPR include: 1) the concept of the environment for cardiac arrest survival and the new chain of survival; 2) the enhanced role of the emergency medical dispatcher in recognizing cardiac arrest and providing CPR through witnesses; 3 ) modification of priority in the maneuvers for managing foreign body airway obstruction; 4) recommendations for the duration of on-site CPR; 5) new recommendations for advanced airway management, drugs, and pediatric defibrillation energy; 6) new recommendations for target temperature management, emergency coronary angiography, neurological prognostication, and rehabilitation of cardiac arrest survivors; and 7) guidelines for CPR for patients with coronavirus disease 2019 (COVID-19) or suspected to have an infection.

\section{Environment for cardiac arrest survival and the new chain of survival}

The environment for cardiac arrest survival includes both medical and non-medical elements in the community that aid the prevention and treatment of cardiac arrest. Since various factors are involved in the process of treating victims of cardiac arrest, a systematic approach is needed to establish an environment for their survival. ${ }^{16}$ Most community members, including government officials, education experts, teachers, students, and the general pub- lic, as well as medical personnel who directly treat patients, should participate in establishing an environment for cardiac arrest survival. In addition, an effective system of care for cardiac arrest, strategies for the prevention of cardiac arrest, and rehabilitation programs for cardiac arrest survivors should be established and implemented. Improvement of the cardiac arrest treatment system can be accomplished by continuous quality assessment and evaluation of system-wide performance. The 2020 CPR guidelines suggest a new chain of survival and the necessity of establishing an environment for cardiac arrest survival. The chain of survival for OHCA consists of early recognition of cardiac arrest and activation of the EMS, bystander (dispatcher-assisted) CPR, defibrillation, ALS, and PCC including rehabilitation. The chain of survival for in-hospital cardiac arrest (IHCA) consists of the detection of early warning signs and a call for a CPR team, high quality CPR, defibrillation, ALS, and PCC including rehabilitation. The environment for OHCA survival includes measures for the prevention of cardiac arrest, dissemination of CPR education, cardiac arrest treatment system, and quality improvement activities. The environment for IHCA survival includes an early warning system and rapid response to IHCA, ALS training for CPR team members, in-hospital system of care, and quality improvement activities.

\section{Enhanced role of emergency medical dispatchers}

Emergency medical dispatchers can help the bystander recognize cardiac arrest and perform CPR. Bystander CPR is associated with increased survival of victims of OHCA. ${ }^{17,18}$ The role of emergency medical dispatchers in the treatment of victims of OHCA is not limited to simply receiving calls and dispatching emergency medical technicians to the scene. The 2020 CPR guidelines recommend that emergency medical dispatchers should assist the bystander to recognize cardiac arrest and perform dispatcher-assisted telephone CPR and should use standard protocols that can help the dispatcher determine whether a cardiac arrest has occurred during an emergency phone call. In addition, the EMS should be equipped with a support system for dispatcher-assisted telephone CPR. Emergency medical dispatchers should be trained to provide the bystander with instructions including directions for dispatcher-assisted telephone CPR.

\section{Changes in BLS}

The 2020 guidelines for the basic techniques of CPR, including CPR sequence, chest compression methods, rescue breathing methods, and compression/ventilation ratio, are the same as the 2015 guidelines for these techniques. The 2020 guidelines recommend that victims should not be moved from places such as beds 
to the floor to improve the depth of chest compressions during CPR. For a lone rescuer with a mobile phone, it is recommended that CPR should be started immediately after turning on the speaker of the mobile phone or activating the handsfree function and seeking assistance from an emergency medical dispatcher if necessary. In the 2020 guidelines, a back blow is recommended as the first maneuver for a victim with foreign body airway obstruction and ineffective coughing effort.

\section{Duration of on-site CPR}

A few observational studies have been conducted to evaluate the effect of the duration of on-scene CPR; however, no large scale randomized study has been conducted on that topic yet. ${ }^{19,20}$ The duration of on-site CPR should be determined considering a variety of factors, including the training level of the emergency medical team providing resuscitation (BLS or ALS level), elapsed time from cardiac arrest, and transport time to the hospital. The 2020 guidelines recommend considering transporting the patient to the hospital if restoration of spontaneous circulation is not achieved after 6 minutes of on-site CPR if the resuscitation team can only perform BLS or after 10 minutes of on-site CPR if resuscitation team can perform ALS.

\section{New recommendations for advanced airway} management, drugs, and pediatric defibrillation energy Regarding the treatment of victims of cardiac arrest, no difference was noted between the resuscitation outcomes of using bag-mask ventilation (BMV) and those of using an advanced airway. ${ }^{21}$ The 2020 guidelines recommend that emergency medical personnel should choose either a bag-mask or an advanced airway (endotracheal intubation or supraglottic airway [SGA]) during resuscitation. However, when the decision to perform advanced airway management is made, it is recommended that emergency medical personnel who lack sufficient training and experience in endotracheal intubation should use the SGA, and only emergency medical personnel with sufficient training and experience may perform endotracheal intubation. An RCT in which amiodarone and lidocaine as antiarrhythmic drugs for the treatment of refractory ventricular fibrillation (VF) were compared with a placebo demonstrated that there was no difference between the resuscitation outcomes of the patients that received amiodarone and those that received lidocaine. ${ }^{22}$ Based on the results of that study, the 2020 guidelines recommend the use of amiodarone and lidocaine equally as antiarrhythmic drugs for the treatment of refractory VF. In the 2020 guidelines for PLS, $2 \mathrm{~J} / \mathrm{kg}$ is recommended as the first defibrillation energy for the treatment of shockable rhythm in children and infants with cardiac arrest.
New recommendations for target temperature management, emergency coronary angiography, neurological prognostication, and rehabilitation of cardiac arrest survivors

The 2020 guidelines recommend that adult victims of non-traumatic OHCA be transferred to a cardiac arrest resuscitation center. A cardiac arrest resuscitation center is a medical institution capable of providing comprehensive PCC, intensive treatment, including 24-hour coronary angiography and target temperature management, and tests for neurological prognostication. Based on the results of a recent trial for victims of OHCA with nonshockable rhythm, the 2020 guidelines recommend performing target temperature management for all victims (irrespective of their initial electrocardiogram rhythm) who are unresponsive after cardiac arrest. ${ }^{23}$ In a study of patients without ST-segment elevation on the electrocardiogram immediately after resuscitation from cardiac arrest, no difference was observed between the resuscitation outcomes of the group that underwent emergency coronary angiography and the group that underwent delayed coronary angiography. ${ }^{24}$ In the 2020 guidelines, emergency coronary angiography is recommended for patients who have ST-segment elevation on the electrocardiogram immediately after resuscitation, patients with cardiogenic shock, or patients with recurrent ventricular arrhythmias.

The 2020 guidelines recommend evaluating the patient to predict neurological outcomes 72 hours after the patient's body temperature returns to normal ( 5 days after restoration of spontaneous circulation). It is recommended that a multimodal approach be used for neurological prognostication based on the results of neurological examination, blood biomarker levels, somatosensory evoked potentials, electroencephalogram, and imaging studies (brain computed tomography scan, brain magnetic resonance imaging). The 2020 guidelines also recommend that a structured screening and assessment of physical and psychological disabilities be conducted and a comprehensive, multidisciplinary discharge plan, including rehabilitation treatment, be established for survivors of cardiac arrest.

\section{Guidelines for CPR for persons with COVID-19 or suspected to have an infection}

Chest compressions during CPR can cause aerosols from the victim to spread through the air. ${ }^{25}$ There is the possibility of airborne transmission of the virus during mouth-to-mouth ventilation, endotracheal intubation, or ventilation with a bag-mask. ${ }^{26}$ Regarding the COVID-19 pandemic, it is more likely that healthcare providers will treat victims of cardiac arrest with COVID-19 or suspected infection. The 2020 guidelines provide guidelines for 
CPR for patients infected with or at risk of infection with COVID-19. The guidelines recommend the use of personal protective equipment to prevent infection, measures to suppress or minimize the formation of aerosols, resuscitation measures to minimize exposure of rescuers to the virus, and treatment algorithms for cardiac arrest, including prioritization of techniques with low aerosol-generating potential.

\section{CONFLICT OF INTEREST}

No potential conflict of interest relevant to this article was reported.

\section{ACKNOWLEDGMENTS}

This study was supported by a grant (2020E330300) of the Korean Disease Control and Prevention Agency funded by the Ministry of Health and Welfare, Republic of Korea.

We thank Ms. So Yeong Kim (EMT) for her assistance with administrative affairs and Mr. Myung Ha Kim for his assistance with literature searches for updating Korean guidelines for CPR. We also thank the Korean Association of Cardiopulmonary Resuscitation (KACPR) for supporting the process of proofreading.

\section{REFERENCES}

1. Berdowski J, Berg RA, Tijssen JG, Koster RW. Global incidences of out-of-hospital cardiac arrest and survival rates: systematic review of 67 prospective studies. Resuscitation 2010;81:1479-87.

2. Korea Disease Control and Prevention Agency. 2006-2018 Cardiac arrest survey [Internet]. Cheongju: Korea Disease Control and Prevention Agency; 2019 [cited 2020 Dec 4]. Available from: http://www.kdca.go.kr/board.es?mid = a20503 050000\&tbid $=0021$ \&tact $=$ view\&list_no $=$ 365592\#quick .

3. Chan PS, McNally B, Tang F, Kellermann A; CARES Surveillance Group. Recent trends in survival from out-of-hospital cardiac arrest in the United States. Circulation 2014;130:1876-82.

4. Stromsoe A, Svensson L, Axelsson AB, et al. Improved outcome in Sweden after out-of-hospital cardiac arrest and possible association with improvements in every link in the chain of survival. Eur Heart J 2015;36:863-71.

5. Kitamura T, Iwami T, Kawamura T, et al. Nationwide improvements in survival from out-of-hospital cardiac arrest in Japan. Circulation 2012;126:2834-43.

6. Song KJ, Kim JB, Kim J, et al. Part 2. Adult basic life support: 2015 Korean Guidelines for Cardiopulmonary Resuscitation. Clin Exp Emerg Med 2016;3(Suppl):S10-6.
7. Guidelines 2000 for cardiopulmonary resuscitation and emergency cardiovascular care. Part 3: adult basic life support. The American Heart Association in collaboration with the International Liaison Committee on Resuscitation. Circulation 2000; 102(8 Suppl):I22-59.

8. International Liaison Committee on Resuscitation. 2005 International Consensus on Cardiopulmonary Resuscitation and Emergency Cardiovascular Care Science with Treatment Recommendations. Part 1: introduction. Resuscitation 2005;67: 181-6.

9. Hazinski MF, Nolan JP, Billi JE, et al. Part 1: Executive summary: 2010 International Consensus on Cardiopulmonary Resuscitation and Emergency Cardiovascular Care Science With Treatment Recommendations. Circulation 2010;122(16 Suppl 2):S250-75.

10. Soar J, Maconochie I, Wyckoff MH, et al. 2019 International Consensus on Cardiopulmonary Resuscitation and Emergency Cardiovascular Care Science With Treatment Recommendations. Resuscitation 2019;145:95-150.

11. Soar J, Donnino MW, Maconochie I, et al. 2018 International Consensus on Cardiopulmonary Resuscitation and Emergency Cardiovascular Care Science With Treatment Recommendations summary. Resuscitation 2018;133:194-206.

12. Atkins DL, de Caen AR, Berger S, et al. 2017 American Heart Association focused update on pediatric basic life support and cardiopulmonary resuscitation quality: an update to the American Heart Association Guidelines for Cardiopulmonary Resuscitation and Emergency Cardiovascular Care. Circulation 2018;137:e1-6.

13. Hwang SO, Chung SP, Song KJ, et al. Part 1. The update process and highlights: 2015 Korean Guidelines for Cardiopulmonary Resuscitation. Clin Exp Emerg Med 2016;3(Suppl): S1-9.

14. Schunemann H, Brozek J, Guyatt G, Oxman A. GRADE handbook [Internet]. London: The Cochrane Collaboration; 2013 [cited 2020 Dec 4]. Available from: https://gdt.gradepro.org/ app/handbook/handbook.html.

15. Morrison $\sqcup$, Gent LM, Lang E, et al. Part 2: evidence evaluation and management of conflicts of interest. 2015 American Heart Association Guidelines Update for Cardiopulmonary Resuscitation and Emergency Cardiovascular Care. Circulation 2015;132(18 Suppl 2):S368-82.

16. Kronick SL, Kurz MC, Lin S, et al. Part 4: systems of care and continuous quality improvement. 2015 American Heart Association Guidelines Update for Cardiopulmonary Resuscitation and Emergency Cardiovascular Care. Circulation 2015;132(18 Suppl 2):S397-413. 
17. Ro YS, Shin SD, Lee YJ, et al. Effect of dispatcher-assisted cardiopulmonary resuscitation program and location of out-ofhospital cardiac arrest on survival and neurologic outcome. Ann Emerg Med 2017;69:52-61.

18. Besnier E, Damm C, Jardel B, Veber B, Compere V, Dureuil B. Dispatcher-assisted cardiopulmonary resuscitation protocol improves diagnosis and resuscitation recommendations for outof-hospital cardiac arrest. Emerg Med Australas 2015;27:590-6.

19. Grunau B, Kime N, Leroux B, et al. Association of intra-arrest transport vs continued on-scene resuscitation with survival to hospital discharge among patients with out-of-hospital cardiac arrest. JAMA 2020;324:1058-67.

20. Kim TH, Lee EJ, Shin SD, et al. Neurological favorable outcomes associated with EMS compliance and on-scene resuscitation time protocol. Prehosp Emerg Care 2018;22:214-21.

21. Benger JR, Kirby K, Black $S$, et al. Effect of a Strategy of a supraglottic airway device vs tracheal intubation during out-ofhospital cardiac arrest on functional outcome: the AIR-
WAYS-2 randomized clinical trial. JAMA 2018;320:779-91.

22. Kudenchuk PJ, Brown SP, Daya M, et al. Amiodarone, lidocaine, or placebo in out-of-hospital cardiac arrest. N Engl J Med 2016;374:1711-22.

23. Lascarrou JB, Merdji $H$, Le Gouge A, et al. Targeted temperature management for cardiac arrest with nonshockable rhythm. N Engl J Med 2019;381:2327-37.

24. Lemkes JS, Janssens GN, van der Hoeven NW, et al. Coronary angiography after cardiac arrest without ST-segment elevation. N Engl J Med 2019;380:1397-407.

25. Nam HS, Yeon MY, Park JW, Hong JY, Son JW. Healthcare worker infected with Middle East Respiratory Syndrome during cardiopulmonary resuscitation in Korea, 2015. Epidemiol Health 2017;39:e2017052.

26. Raboud J, Shigayeva A, McGeer A, et al. Risk factors for SARS transmission from patients requiring intubation: a multicentre investigation in Toronto, Canada. PLoS One 2010;5:e10717. 